\title{
Efficacy of Transarterial Chemoembolization Combined with Radiofrequency Ablation in Treatment of Hepatocellular Carcinoma
}

\author{
Chuan Xu1 ${ }^{1}$, Peng-Hua Lv ${ }^{1 *}$, Xin-En Huange*, Shu-Xiang Wang ${ }^{1}$, Ling Sun ${ }^{1}$, \\ Fu-An Wang ${ }^{1}$
}

\begin{abstract}
Purpose: To evaluate efficacy of transarterial chemoembolization (TACE) combined with radiofrequency ablation (RFA) in treatment of patients with hepatocellular carcinoma. Materials and Methods: During January 2009 to March 2012, 80 patients with hepatocellular carcinoma underwent TACE, with or without RFA. Alfafetoprotein (AFP) was checked before and after procedure. CT scans were obtained one month after TACE or RFA for all patients to evaluate tumor changes. Complete response+partial response+stable disease $(\mathrm{CR}+\mathrm{PR}+\mathrm{SD}) / \mathbf{n}$ were used to assess the disease control rate (DCR). Survival at 3,6 and 12 months was compared in both groups. Results: AFP levels in TACE + RFA group dropped rapidly, becoming obviously lower than that of the TACE group. In the TACE + RFA group DCR was $93.8 \%$, while only $76.8 \%$ in the TACE group. The treatment effect between the two groups was statistically significant $(P<0.05)$ by Ridit analysis. 1 year survival rate in the TACE + RFA group was $92.5 \%$, significantly higher than that of the TACE group at $77.5 \%(P<0.05)$. Conclusions: TACE and RFA as combined therapy method for patients with middle and terminal stage HCC gives full play to synergy between the two and improves the therapeutic effect.
\end{abstract}

Keywords: Transarterial chemoembolization - radiofrequency ablation - combination - hepatocellular carcinoma

Asian Pac J Cancer Prev, 16 (14), 6159-6162

\section{Introduction}

Hepatocellular carcinoma (HCC) is the sixth most common cancer in the world and the third most common cause of cancer-related death (Benson et al., 2009). Hepatic resection is still to get the possibility of cure for patients with $\mathrm{HCC}$, but majority of patients diagnosed with HCC have loose surgery opportunity (Hsu et al., 2011). Transarterial chemoembolization (TACE) is used frequently for the treatment of HCC when other curative treatment are not possible(Wilson et al., 2012). In real clinical practice, repeated TACE procedures are often needed, since the best response cannot always be achieved after one session of TACE (Golfieri et al., 2013).

Radiofrequency ablation (RFA) is emerging as an effective local treatment for HCC smaller than $3 \mathrm{~cm}$ in diameter. Survival of patients with HCC less than $3 \mathrm{~cm}$ treated by RFA competes with that of surgical candidaties(Yau et al., 2014). Both methods are effective in treatment of HCC. But in clinical practice, the recurrence rate is higher in patients received TACE and intrahepatic recurrence rates were up to 60 percent have been reported. Some studies combined TACE with RFA to treat $\mathrm{HCC}$ received a good prognosis(Wilson et al., 2012). Because TACE is a safe and effective alternative to hepatic resection and FRA for $\mathrm{HCC}$ of $3 \mathrm{~cm}$ or smaller without vascular invasion especially for patients who are vulnerable to adverse events of treatments.

\section{Materials and Methods}

We retrospectively analysis 80 patients (man 53; woman 27 ; mean age $63 \pm 14$ ) with histologically proven primary HCC underwent TACE with $(\mathrm{N}=)$ or without $(\mathrm{N}=)$ RFA in January 2009 to March 2012. All patients were considered to have unresetable disease based on the number of bilobar distribution of lesions, involvement of major vascular structures precluding curative resection, or inadequate reserve to undergo resection. The patients' characteristics are summarized in Table 1 . There was no radiographic evidence of extrahepatic disease. Patients with HCC were treated with TACE alone and TACE combination with RFA. All patients provided written, informed consent for the procedure, and our institutional review board approved the retrospective review of the patients' medical and imaging records.

TACE

Vascular access was obtained via the right common femoral artery and a guidewire was advanced under

${ }^{1}$ Department of Interventional Radiology, Subei People's Hospital of Jiangsu Province, Clinical Hospital of Yangzhou University, Yangzhou, ${ }^{2}$ Department of Chemotherapy, Jiangsu Cancer Hospital, Nanjing Medical University, Nanjing, Jiangsu, China *For correspondence: why777sina.com; huangxinen06@163.com 
Table 1. Demopraphics of Patients Undergoing TACE with or without RFA of HCC

\begin{tabular}{lll}
\hline & \multicolumn{1}{c}{ TACE } & TACE+RFA \\
\hline $\mathrm{N}$ & 42 & 38 \\
Men & 28 & 25 \\
age & $59.3 \pm 10.4$ & $60.1 \pm 9.3$ \\
\hline
\end{tabular}

Table 2. AFP Levels Change between TACE+RFA and TACE before and After Procedure (ng/L)

\begin{tabular}{lrcc}
\hline & Before & 1 Week After & One Month After \\
Procedure & Procedure & Procedure \\
\hline TACE+RFA & $244.6 \pm 3.3$ & $139.3 \pm 1.8$ & $113.0 \pm 1.5$ \\
TACE & $246 \pm 2.0$ & $199.5 \pm 2.1$ & $173.5 \pm 4.7$ \\
P & 0.51 & 0.007 & 0.004 \\
\hline
\end{tabular}

fluoroscopic guidance. A 5-F sheath was then inserted over the guidewire. Common hepatic and superior mesenteric arteriographies were initially performed to assess patient anatomy, tumor burden, vascularity, and portal vein patency. Once the vascular supply of the tumor was identified chemoembolization of the supplying artery was undertaken. Lipiodial and Epirubicin hybrid emulsion was infused into the selected segmental feeding artery until arterial flow stasis achieved. Successful embolization of the feeding vessel was confirmed by angiogram. The catheter and wire were then removed and direct pressure was held for 20 minutes.

\section{RFA}

Patients were considered for RFA regardless of proximity of the lesions to major portal or hepatic vein branches. RFA was preferentially undertaken percutaneously utilizing CT scan guidance. RFA was performed by using Therapeuties RF2000 system (Mountain View, Calif) in this study. Once in place power is applied by the RF2000 generator, which can deliver power up to $100 \mathrm{~W}$. Power was increased in a stepwise fashion beginning at $50 \mathrm{~W}$ until maximum power was reached. Tumor ablation was continued at maximum power until tissue impedance increased to the point when power output fell rapidly (i.e., "roll-off"). If rolloff was unable to be achieved ablation was continued at maximum power for 15 minutes. Ablation was carried out a second time utilizing a similar stepwise increase in power until maximum power output was achieved. Ablation was again continued until roll-off for 10 minutes. Small tumors $(<3 \mathrm{~cm})$ were ablated after a single passage of the electrode array into the center of the lesions. For larger tumors the electrode array was repositioned at $3-\mathrm{cm}$ interval and ablation carried out as above so as to allow complete destruction of the tumor with a one-cetimeter margin. 117 lesions were treated by RFA in 80 patients, all of them were routine to protect liver, acid suppression and support treatment.

\section{Follow-up}

CT scans were obtained one month after TACE or RFA for all patients and evaluated the tumor changes. The standard of evaluation are follows: 1, complete response (CR): the tumor disappeared, all tumor foci were inactive;
Table 3. Survival Rates between TACE+RFA and TACE Group

\begin{tabular}{lccc}
\hline & 3 months & 6 months & 1 year \\
\hline TACE+RFA $(\%)$ & 100 & 97.5 & 92.5 \\
TACE $(\%)$ & 96.9 & 85.7 & 77.5 \\
P & 0.64 & 0.023 & 0.017 \\
\hline
\end{tabular}

2, partial response $(\mathrm{PR})$ : total diameter of tumor active part reduce $>30 \% ; 3$, stable disease (SD): total diameter of tumor active part reduce $<30 \%$ or increase $>20 \% ; 4$, progressive disease (PD): the total diameter of tumor active part increase $>30 \%$. And defining $(\mathrm{CR}+\mathrm{PR}+\mathrm{SD}) / \mathrm{n}$ to count the disease control rates (DCR).

\section{Statistical analysis}

All statistical analyses were performed by using SPASS17.0 and $P$ value less than 0.05 was considered to indicate a significant. The measurement data were compared by using t test, Ridit analysis was used to the curative effect of hierarchical data difference, and KaplanMeier method is used to test on survival.

\section{Results}

42 patients underwent TACE alone and 38 patients received TACE and RFA. Comparing AFP levels change between TACE+RFA and TACE before and after procedure. The two groups had no obvious difference were found before procedure. One week and one month after procedure, AFP levels in TACE and RFA group drop rapidly, obviously lower than that of TACE group (Table 2). In TACE and RFA group DCR was $93.8 \%$, while $76.8 \%$ in TACE group. The treatment effect between the two groups was statistically significant $(P<0.05)$ by Ridit analysis.

Two group of patients survival rate comparison: 1 year survival rates in TACE and RFA group was $92.5 \%$ $(37 / 40)$, significantly higher than that of TACE group $77.5 \%$ (31/40), have statistically significant $(P<0.05)$. But three months survival rate has no statistical significance $(P>0.05)$.

\section{Discussion}

Early diagnosis for patients with HCC is difficult, most of them are diagnosed with moderate or advanced and surgical resection rate is very low. TACE is one of the first choices for patients curative treatment are not possible (Cho et al., 2010). TACE is injecting embolic materials into arteries supply tumor by catheter, leading tumor lack of ischemia and necrosis (Germani et al., 2010). At the same time, anti-tumor drug was brought to local tumor and release slowly, killing tumor cells for a long time (Yang et al., 2012). In clinical practice, we found that tumor necrosis rate is low for patients with $\mathrm{HCC}$ received TACE, and the recurrence rate is high (Burrel et al., 2012). Main reasons are follow: 1, Peripheral blood supply of tumor from the portal vein; 2, Establishment of collateral circulation after TACE; 3 , There may be many arterial blood supply. 4, Incomplete embolism. 5, Patients with 
HCC received TACE could induce vascular endothelial growth factor which could promote vascular growth factor expression, increasing the risk of tumor recurrence and distant metastasis (Llovet et al., 1999; Bruix et al., 2001). And TACE has limitations, may not apply to all patients with liver function status, only the Child - Pugh grade $\mathrm{A}$ or $\mathrm{B}$ of the patients can be implemented. RFA is a physical ablation treatment for HCC, which is by the guidance of imaging technologies, including ultrasound, $\mathrm{CT}$ and so on. The needle was directly percutaneous into the tumor, and using high frequency current in vivo tissue ion with the current changes in the direction of vibration, making organization around the electrode current friction to generate heat, so that the local tissue protein denaturation, membrane disruption, coagulation necrosis and carbonization, achieve the goal of treatment of tumor.

In 2005, RFA as a means for the treatment of liver cancer in American Association for the Study of Liver Diseases. The main indications were: 1 , Unresectable liver cancer, such as tumor located in liver lobes or particularly big, combined with liver cirrhosis at the same time, the residual liver will not be able to meet the body's physiological needs after surgical removal; 2, Waiting for liver transplantation; 3 , Recurrence after resection of liver cancer; 4, resectable liver cancer, but Patients are reluctant to accept surgery (Liapi et al., 2011). For patients with moderate or advanced liver cancer may lead to incomplete ablation and ultimately affect the effect of the treatment. Some scholars think that radiofrequency ablation has a unique advantage compared to surgical treatment: 1, radiofrequency ablation could puncture into deeper tumor which cannot be resected; 2, radiofrequency ablation has less effect on normal liver tissue and liver function damage with small; 3 , compared to traditional laparotomy, radiofrequency ablation is a minimally invasive surgery and lower incidence of complications; 4, some studies found that radiofrequency ablation can cause tumor cell necrosis which could cause specific cellular immunity and prevent tumor recurrence (Riaz et al., 2010; Yau et al., 2014). But when the lesion is more than $5 \mathrm{~cm}$, RFA therapy is difficult to achieve complete necrosis. RFA therapy complications may higher when the lesion located under the diaphragm or near the portal vein or inferior to vena cava and its application is suppressed (Golfieri et al., 2013). Both TACE and RFA have advantages and disadvantages, and combination of them, could achieve the complementary advantages.

We check AFP one week and one month after procedure, AFP levels in TACE and RFA group drop rapidly, obviously lower than that of TACE group. Some studies have found that DCR in TACE and RFA group was about $93.7 \%$, while TACE only treatment was $43.8 \%$ (Reig et al., 2013). In our study, DCR in TACE and RFA group was $93.8 \%$ and $76.8 \%$ in TACE group $(P<0.05)$, which have statistical significance. 1 year survival rate was 92.5\% in TACE and RFA group, significantly higher than that of TACE group which was $77.5 \%$. The recurrence rate is low if combined TACE and RFA to treat patient with HCC, 6 months and 1 year survival rate is higher, because TACE and RFA could achieve complementary advantages. First of all, tumor blood vessels can be embolized taken
TACE, reduce tumor blood flow, reduce the heat loss effect when receive RFA, increase the ablation range. Second, tumor foci reduced after TACE, and Lipiodial deposit in the tumor which making accurate positioning and precise ablation for tumor (Chinn et al., 2001; Yau et al., 2014).

TACE and RFA are all minimally invasive treatment, patients recover quickly, can be repeated, more likely to be accepted by patients compared to surgery. Combination with our results, we recommend TACE and RFA as combined therapy method for patients with middle and terminal stage HCC, give full play to the synergy of the two, and improve the therapeutic effect.

\section{References}

Benson AB, Abrams TA, Ben JE, et al (2009). NCCN clinical practice guidelings in omcology: hepatobiliary cancers. $J$ Natl Compr Canc Netw, 7, 350-59.

Bruix J, Sherman M, Llovet JM, et al (2001). Clinical management of hepatocellular carcinoma: conclusions of the Barcelona-2000 EASL conference. European Association for the study of the Liver. $J$ Hepatol, 35, 421-30.

Burrel M, Reig M, Forner A, et al (2012). Survival of patients with hepatocellular carcinoma treated by transarterial chemoembolization (TACE) using Drug Eluting Beads. Implications for clinical practice and trial design.J Hepatol, 56, 1330-35.

Chinn SB, Lee FT, Kennedy, et al (2001). Effect of vascular occlusion on radfiofrequency ablation of the liver: Results in a porcine model. AJR Am J Roentgenol, 176, 789-95.

Cho YK, Kim JK, Kim WT, at al (2010). Hepatic resection versus radiofrequency ablation for very early stage hepatocellular carcinoma: a Markov model analysis. Hepatol, 51, 1284-90.

Germani G, Pleguezuelo M, Gurusamy, et al (2010). Clinical outcomes of radiofrequency ablation, percutaneous alcohol and acetic acid injection for hepatocellular: a meta-analysis. J Hepatol, 52, 380-87.

Golfieri R, Renzulli M, Mosconi C et al (2013). Hepatocellular carcinoma responding to superselective transarterial chemoembolization: an issue of nodule dimension? J Vasc Interv Radiol, 24, 509-17.

Hsu CY, Huang YH, Chiou YY, et al (2011). Comparision of radiofrequency ablation and transterial chemoembolization for hepatocellular carcinoma within the Milan criterial: a propensity score analysis. Liver Transpl, 17, 556-66.

Liapi E, Geschwind JF (2011). Transcatheter arterial chemoembolization for liver cancer: is it time to distinguish conventional from drugeluting chemoembolization? Cardiovasc Intervent Radiol, 34, 37-49.

Llovet JM, Bru C, Bruix J (1999). Prognosis of hepatocellular carcinoma: the BCLC staging classification. Semin Liver Dis, 19, 329-38.

Riaz A, Lewandowski RJ, Kulik L, et al (2010). Radiologicpathologic correlation of hepatocellular carcinoma treated with chemoembolization. Cardiovasc Intervent Radiol, 33, 1143-52.

Reig M, Rimola J, Torres Fet al (2013). Postprogression survival of patients with advanced hepatocellular carcinoma: rational for second-line trial design. Hepatol, 2023-31

Wilson TR, Fridlyand J, Yan Y, et al (2012). Widespread potential for growth-factor-driven resistence to anticancer kinase inhibitors. Nature, 487, 505-9.

Yang JD, Kim WR, Park KW, et al (2012). Model to estimate survival in ambulatory patients with hepatocellular carcinoma. Hepatol, 56, 614-21.

Yau T, Tang VY, Yao TJ, at al (2014). Development of 
Chuan Xu et al

Hong Kong liver cancer staging system with treatment stratification for patients with hepatocellular carcinoma. Gastroenterol, 146, 1691-170. 